\title{
Reformas constitucionales $y$ competencia política en Bolivia
}

\author{
Mario Torrico
}

Profesor - investigador de la FLACSO, Sede México

\begin{abstract}
This article explains the causes and effects of institutional change in political contexts where there is no guarantee of strict compliance with the rules. Analyzing the political reform of 2005 that made it possible for prefects or governors to be elected for the first time in Bolivia by popular vote, evidence was found that when there is a crisis of legitimacy, declining coalitions push for inclussive changes that reduce future electoral losses and cannot be reversed by the rising coalitions that come to power. These coalitions, nonetheless, attempt to limit the degree of inclusion of rules through lowerlevel policy changes that reduce political competition.

\section{Keywords}

changes in the subnational electoral system, democratic continuity, electoral reforms, neoinstitutionalism, prefectural elections, subnational political competence, 2005 political reform
\end{abstract}




\section{Resumen}

El presente artículo explica las causas y efectos del cambio institucional en contextos políticos en los que no hay garantía sobre el cumplimiento estricto de las reglas. Analizando la reforma política de 2005 que posibilitó (por primera vez) que en Bolivia los prefectos o gobernadores puedan ser elegidos por voto popular, se halla evidencia que indica que cuando hay crisis de legitimidad, las coaliciones declinantes impulsan cambios incluyentes que reducen sus futuras pérdidas electorales y no pueden ser revertidos por las coaliciones ascendentes que llegan al poder. No obstante, éstas intentan limitar el grado de inclusión de las reglas a través de cambios normativos de menor nivel que disminuyen la competencia política.

\section{Palabras claves}

cambios en el sistema electoral subnacional, competencia política subnacional, continuidad democrática, elecciones prefecturales, neoinstitucionalismo, reforma política de 2005, reformas electorales

\section{Introducción}

La democracia en Bolivia lleva más de tres décadas de existencia ininterrumpida, algo inédito en la historia de un país que acumula 192 golpes de Estado a lo largo de su historia. No obstante, la continuidad democrática no ha estado exenta de fuertes tensiones, y para sortearlas se ha tenido que organizar elecciones presidenciales anticipadas, lograr acuerdos extra institucionales entre los principales actores políticos, e incluso reformar parcial y totalmente la Constitución. Esas acciones resultaron inevitables debido a determinadas coyunturas críticas y a cambios en el contexto político. Por ejemplo, a raíz de que el gobierno de Hernán Siles Zuazo no pudo contener la peor crisis económica de la historia ni evitar el conflicto social resultante, el presidente, con la mediación de la jerarquía de la Iglesia Católica, acordó con los demás partidos políticos el adelantamiento de las elecciones que debían efectuarse en 1986, realizándose las mismas en 1985. Puesto que la Constitución no contemplaba siquiera la posibilidad de elecciones anticipadas, esta salida negociada claramente no se ajustó a las opciones jurídicamente posibles, pero logró preservar la democracia ante la amenaza de que los militares regresen al poder a través de un nuevo golpe.

Durante más de veinte años la competencia política boliviana se dio casi exclusivamente en el ámbito de las elecciones presidenciales. En un Estado 
unitario en el que las máximas autoridades departamentales (los prefectos, ahora llamados gobernadores) eran nombradas directamente por el presidente, y en el que la composición del Congreso se realizaba a través de listas cerradas y bloqueadas que presentaban los partidos en cada elección presidencial, las dinámicas subnacionales eran poco relevantes. A pesar de que a partir de 1987 existen elecciones para alcaldes y concejales en los municipios, sólo se podía postular a través de algún partido político con registro nacional (no existían partidos de índole local), lo que provocaba que las dinámicas nacionales se impusieran permanentemente a las locales. En ese sentido, la actividad política municipal o departamental era vista por los políticos sólo como un escalón en una carrera que aspiraba siempre a construir un liderazgo nacional.

A comienzos de este siglo Bolivia atravesó por una recesión económica provocada por los efectos negativos de las crisis brasileña y argentina, que ocasionó que el crecimiento por habitante entre 1999 y 2003 fuera prácticamente nulo $(0,08$ por ciento), y también enfrentó una fuerte crisis política que provocó la renuncia y huida del presidente Gonzalo Sánchez de Lozada en 2003. Carlos Mesa, quien fuera su vicepresidente, asumió la Presidencia, pero sólo duró poco más de un año en el poder y se tuvo que convocar a elecciones anticipadas en 2005 (el periodo de gobierno finalizaba en 2007), las que ganó Evo Morales. De forma simultánea a la elección presidencial, se realizaron por primera vez elecciones de prefectos departamentales. Desde entonces, y ya en el marco de la Nueva Constitución aprobada en 2009, se han producido otros dos procesos electorales para Presidente y para gobernadores. ¿A qué se debió la reforma que permitió que los prefectos sean electos? ¿Por qué ese cambio se mantuvo en la Nueva Constitución? ¿Qué transformaciones se han producido en la competencia política a partir de entonces? Estas son las preguntas que se abordarán en el presente artículo.

\section{2. ¿Por qué cambian las reglas?}

La literatura sobre elección nacional señala que los actores persiguen sus objetivos a través del desarrollo de estrategias en función del marco institucional existente o del cambio de dicho marco, lo que a su vez transforma las estrategias de todos los actores (Tsebelis). En ese sentido, el cambio en las reglas ocurre cuando los resultados que arrojan no son satisfactorios para los actores políticos relevantes o cuando ha habido alguna transformación en el 
contexto en el que operan las instituciones que permite anticipar que los futuros resultados no serán satisfactorios. Ahora bien, para el neoinstitucionalismo las reglas constituyen restricciones al comportamiento (North) debido a que asume implícitamente que el Estado puede garantizar su cumplimiento y que no cambian frecuentemente. Ello significa que todos pueden dar por sentado que una vez establecidas van a durar mucho tiempo y programar sus acciones futuras en consecuencia. Si ambos supuestos no se cumplen, los individuos no orientarán sus acciones en función de las instituciones.

La experiencia en América Latina respecto a las reglas que tienen efectos sobre la competencia política nos muestra que sí se puede asumir su cumplimiento (para lo cual ha sido muy importante la creación de órganos electorales especializados que generen confianza en torno a los resultados electorales) pero no así su estabilidad en el tiempo. Por ejemplo, en la década del ochenta era predominante que se elijiera a los presidentes por mayoría simple y que no hubiera posibilidad de reelección inmediata, pero a inicios de este siglo el método de mayoría absoluta con segunda vuelta y la aprobación de una reelección inmediata estaban ya contemplados en las constituciones de la mayoría de los países de la región. En Colombia esta inestabilidad institucional ha sido incluso mayor, ya que en 2005 se impulsó un cambio constitucional para permitir la reelección inmediata del Presidente, pero en 2015 se la prohibió nuevamente. Brasil está a punto de hacer lo mismo: en 1997 aprobó la reelección presidencial inmediata y en 2015 la Cámara de Diputados votó para prohibirla. Actualmente esa disposición está en espera de ser tratada en el Senado, y todo apunta a que será aprobada.

La experiencia con las reglas institucionales en América Latina nos muestra que los supuestos que maneja el neoinstiucionalismo son en realidad variables, y que el comportamiento de los actores puede modificarse en función de ello (Levitsky y Murillo). Si el Estado no está en condiciones de garantizar el cumplimiento de las reglas y si éstas cambian constantemente, es esperable que las acciones de individuos y de grupos no se ajusten a lo que se pretende con las normas. Este señalamiento se aleja notablemente de planteamientos clásicos de la Ciencia Política, como los de Duverger o de Cox, para quienes las reglas generan efectos claros y predecibles sobre la competencia política. ¿Esto significa que no podemos hacer generalizaciones al respecto? No del todo. Incluso en condiciones de poca fortaleza institucional las reglas tienen algún grado de efecto, de lo contrario los actores no buscarían cambiarlas. Eso expresa que aún en países en que su cumplimiento no está garantizado, es relevante entender por qué se las cambia. En un artículo 
titulado "Entre la legitimidad y la eficacia: Reformas en los sistemas de elección presidencial en América Latina", Daniel Buquet propone una teoría para explicar el cambio institucional que parte de la identificación de déficits de legitimidad o de eficacia del gobierno. Cuando se produce alguno de los fenómenos mencionados surgen dos clases posibles de reforma que dependen del tipo de coalición dominante que la promueva: declinante o ascendente. Una coalición declinante está formada por viejos partidos ganadores que se perciben como futuros perdedores y buscan minimizar las ulteriores pérdidas a través de reglas más incluyentes, por ejemplo, disminuyendo el umbral electoral o aplicando una fórmula de representación proporcional en sustitución de una de mayoría simple para integrar el Congreso. En cambio, una coalición ascendente es aquella que se beneficia del cambio en las preferencias electorales y busca maximizar sus futuras ganancias a través de un diseño más excluyente, por ejemplo, ampliando los años en funciones de las autoridades electas o permitiendo la reelección inmediata cuando no estaba permitida.

En este trabajo se pondrá a prueba la propuesta teórica de Buquet pero en un contexto distinto para el cual fue pensado: la competencia política subnacional. Ahora bien, no puede ignorarse que aún cuando las reglas se diseñan con una intencionalidad clara, no se elimina la incertidumbre sobre su efecto final, algo con lo que se debe lidiar en todo proceso de reforma política (Jung y Deering), con el agravante de que una vez que las nuevas reglas estén funcionando será muy difícil enmendarlas o eliminarlas, incluso si el nuevo diseño arroja resultados perjudiciales para los actores dominantes (McFaul). Es decir, las nuevas reglas empiezan a reconfigurar las preferencias de todos los actores y la distribución del poder desde un inicio, de tal manera que dar marcha atrás podría generar conflictos importantes (se genera una dependencia de la trayectoria o path dependence). Sin duda la incertidumbre es mayor en contextos en que no hay garantía sobre el cumplimiento estricto de las reglas, como ocurre en países con democracias aún en proceso de consolidación.

Con base en lo mencionado en los dos párrafos anteriores se someterá a prueba la siguiente afirmación: la reforma que permitió que los prefectos/gobernadores sean electos fue impulsada por una coalición política declinante que, debido a problemas de legitimidad, vislumbraba una futura derrota y buscaba minimizar sus pérdidas. Una vez que esa regla entró en funcionamiento el nuevo actor dominante no tuvo posibilidad de eliminarla, teniendo como efecto que en Bolivia haya mayor competencia política a nivel subnacional de la que existe a nivel nacional y que haya mayor pluralidad en 
los gobiernos departamentales de la que había bajo la regla anterior, cuando el presidente nombraba a los prefectos. Esto último significa que, a pesar de la incertidumbre existente en el momento del diseño de la nueva regla, los objetivos que la coalición declinante buscaba al respecto se cumplieron.

\section{Legitimidad y reforma política}

Durante casi veinte años los actores centrales de la competencia política en Bolivia fueron tres partidos: el MNR (Movimiento Nacionalista Revolucionario), ADN (Acción Democrática Nacionalista y MIR (Movimiento de Izquierda Revolucionaria). Entre los tres concentraban, a mediados de los ochenta, casi tres cuartas partes de los votos, pero su importancia electoral fue paulatinamente disminuyendo elección tras elección hasta que desaparecieron la década pasada, lo que podría ser interpretado como un problema de legitimidad que no pudieron superar.

Un aspecto importante en las elecciones presidenciales hasta antes de 2009 era que la Constitución establecía que, si ningún candidato obtenía la mayoría absoluta de los votos, el Congreso elegiría al presidente; es decir, la segunda vuelta era legislativa. Ello obligaba a que los partidos formasen alianzas post-electorales que luego se convertían en coaliciones de gobierno, ${ }^{1}$ pero además incentivaba a que se presentasen muchos candidatos a elecciones, ya que cada uno intentaba obtener fuerza electoral suficiente que le permita negociar posteriormente espacios en el gobierno. Eso dio como resultado que Bolivia tenga un sistema de partidos altamente fragmentado. ${ }^{2}$

Debido a que era típico que el electorado divida su voto entre las diferentes candidaturas, entre 1985 y 2002 prácticamente ningún partido pudo gobernar por sí solo. Además, dado que la fragmentación de los votantes creció sostenidamente, las coaliciones de gobierno tuvieron que incluir a más partidos, lo que dificultaba la efectividad y la oportunidad en la toma de decisiones. Ello también ocasionó que los incentivos para la extracción de rentas de los partidos gobernantes aumenten, ya que los espacios de poder que les tocaban eran cada vez menores. Así, los escándalos de corrupción se multiplicaron a finales de los noventa y a inicios de este siglo. En la siguiente Tabla se puede observar la evolución de lo hasta aquí mencionado. (Fig. 1)

\footnotetext{
${ }^{1}$ Véase el trabajo de Blithz Lozada y Marco Antonio Saavedra citado en la bibliografía.

2 Más detalles sobre dichos incentivos se encuentran en Mario Torrico (2014).
} 


\begin{tabular}{|c|c|c|c|c|}
\hline $\begin{array}{c}\text { Año de } \\
\text { elecciones }\end{array}$ & $\begin{array}{c}\text { Núm. Partidos } \\
\text { participantes }\end{array}$ & $\begin{array}{c}\text { Porcentaje } \\
\text { del ganador }\end{array}$ & $\begin{array}{c}\text { MNR+ADN } \\
\text { +MIR }\end{array}$ & $\begin{array}{c}\text { Partidos en el } \\
\text { gobiemo }\end{array}$ \\
\hline 1985 & 18 & $32.83 \%$ & $73.37 \%$ & MNR, ADN \\
\hline 1989 & 10 & $25.64 \%$ & $72.64 \%$ & MIR, ADN \\
\hline 1993 & 14 & $33.84 \%$ & $53.87 \%$ & MNR,UCS,MBL \\
\hline 1997 & 10 & $22.30 \%$ & $57.30 \%$ & $\begin{array}{c}\text { ADN, MIR, } \\
\text { CONDEPA, UCS }\end{array}$ \\
\hline 2002 & 11 & $22.50 \%$ & $42.20 \%$ & MNR, MIR, UCS \\
\hline 2005 & 8 & $53.72 \%$ & $6.46 \%$ & MAS \\
\hline 2009 & 8 & $64.20 \%$ & - & MAS \\
\hline 2014 & 5 & $61.36 \%$ & - & MAS \\
\hline
\end{tabular}

Figura 1: Indicadores en elecciones presidenciales en Bolivia Fuente: elaboración propia

La fragmentación del sistema de partidos y del voto desaparecieron en 2005, cuando Evo Morales ganó las elecciones por mayoría absoluta y pudo gobernar sin necesidad de construir una coalición de gobierno. Desde entonces el MAS (Movimiento al Socialismo) ha sido el actor político dominante ganando las posteriores elecciones presidenciales con más del 60 por ciento de los votos, y el anterior sistema de partidos ha desaparecido. ${ }^{3}$ Ello indica que los partidos y el propio sistema político sufrieron una crisis de legitimidad de la que no pudieron recuperarse y que fue capitalizada por el MAS. Los gráficos a continuación así lo confirman. (Figs. 2 y 3)

\footnotetext{
${ }^{3}$ Un factor adicional que contribuyó al éxito del MAS tiene que ver con el fuerte apoyo electoral que recibió Evo Morales de los electores indígenas, en general, y de los quechuas y aymaras en particular. Como se sabe, Bolivia es un país con una población indígena muy grande (al menos del 41 por ciento) en la que ambos grupos son los más numerosos y por primera vez en 2005 concentraron su voto en un candidato. Así, el 76 por ciento de quienes se identificaron como indígenas reportó haber votado por el MAS en esa elección, en comparación con el 55 por ciento de mestizos y el 34 por ciento de blancos. De entre los indígenas, el 72 por ciento de quechuas y el 81 por ciento de aymaras mencionaron que su voto fue para Evo Morales (según datos de LAPOP). Esto muestra que en 2005 la identidad indígena se convirtió por primera vez en un activo electoral (en 2002 sólo el 22 por ciento de indígenas había votado por Evo Morales, quien de manera sorpresiva obtuvo el segundo lugar en la elección presidencial). Hay amplia bibliografía que desde la sociología aborda este aspecto, destacando los trabajos de Alvaro García Linera, Hervé Do Alto y Maristella Svampa (todos citados en la bibliografía).
} 


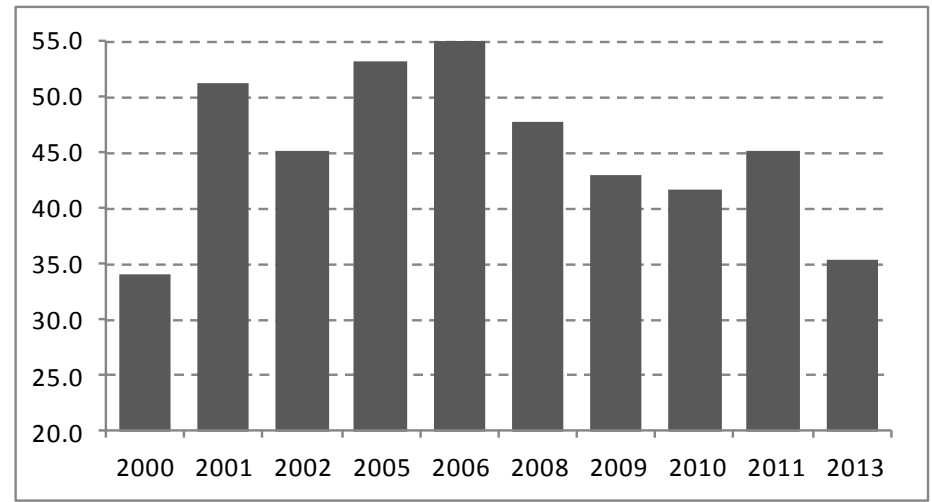

Figura 2: Porcentaje de personas que consideran que "la democracia puede funcionar sin partidos" 4

Fuente: elaboración propia con datos de Latinobarómetro

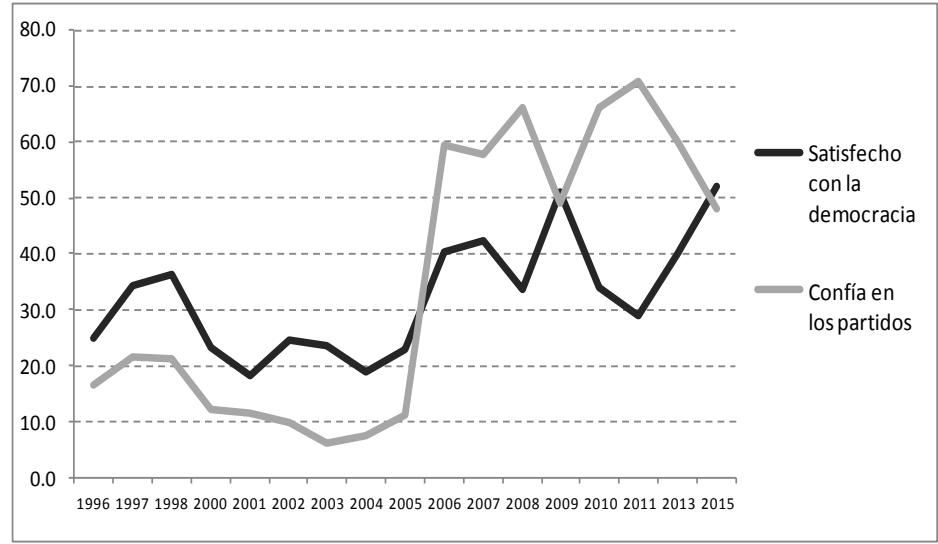

Figura 3: Porcentaje de personas "satisfechas con la democracia" y que "confían en los partidos"

Fuente: elaboración propia con datos de Latinobarómetro

\footnotetext{
${ }^{4}$ En los años 2003, 2007 y 2015 la encuesta no incluyó esa pregunta.
} 
En ambos gráficos se puede observar que la opinión negativa hacia los partidos alcanzó niveles críticos entre 2001 y 2005. En 2006, año en que empezó el primer gobierno de Evo Morales, la confianza hacia ellos aumentó notablemente (lo que se debe a la imagen positiva del Presidente), pero la mayoría de las personas aún opinaba que éstos no eran indispensables para la democracia. Así también, la satisfacción con la democracia presentó sus niveles más bajos entre 2001 y 2005 . Luego de esa fase crítica la imagen de los partidos y de la democracia repuntó notablemente, cuando el sistema de partidos desapareció, el MAS ya dominaba en la escena política nacional boliviana, y la competencia política en elecciones presidenciales había disminuido. Ello evidencia que tanto los partidos previamente dominantes como el sistema político atravesaron a inicios de este siglo una crisis de legitimidad que les resultó insuperable. En consecuencia, en esos años se presentó una de las condiciones que según Buquet motivan reformas políticas de cambio institucional: un déficit de legitimidad.

De acuerdo a la Constitución de 1967, en Bolivia los partidos políticos tenían el monopolio de la representación, lo que fue ratificado por la Ley de Partidos Políticos de 1999 que señalaba: "la representación popular se ejerce por medio de los partidos políticos y las alianzas formadas por éstos" (artículo 4). Ante los problemas de confianza y credibilidad que atravesaron los mismos a principios de la década pasada, y luego de la renuncia y auto exilio de Sánchez de Lozada, en julio de 2004 se aprobó la Ley de Agrupaciones Ciudadanas y Pueblos Indígenas, que autorizó a ese tipo de organizaciones a participar de los procesos electorales y presentar candidatos propios. Dicho ordenamiento jurídico estipulaba: "la intermediación de la representación popular se ejerce a través de los Partidos Políticos, Agrupaciones Ciudadanas y Pueblos Indígenas con registro por el Órgano Electoral". (artículo 6)

Pocos meses después, en enero de 2005, el presidente Carlos Mesa emitió el Decreto Supremo 27988 que convocaba a elecciones para la selección de prefectos en todos los departamentos del país, elecciones en las que podían participar los partidos políticos, las agrupaciones ciudadanas y los pueblos indígenas. En sus consideraciones, el mencionado decreto señalaba: "Bolivia está viviendo un proceso de transición histórica en el cual resulta de primordial importancia profundizar la Descentralización Administrativa. En ese propósito es necesario avanzar con medidas concretas que acerquen al ciudadano a la toma de decisiones en el ámbito Departamental". Esta frase refleja que en el gobierno había plena consciencia del déficit de legitimidad que se vivía en ese momento. 
La convocatoria a elecciones prefecturales fue modificada dos veces, en virtud de omisiones legales en el Decreto Supremo 27988. Debido a la inestabilidad política de ese periodo las tres convocatorias fueron firmadas por dos presidentes de la República y un presidente del Senado: la primera por Carlos Mesa, la segunda por Hormando Vaca Díez (presidente del Senado) y la tercera por Eduardo Rodríguez (las últimas a través de los decretos supremos 28077 y 28229 , respectivamente). Además, se tuvieron que emitir dos leyes: 1 ) una ley interpretativa de la Constitución que establecía que la designación presidencial de los prefectos de Departamento sería precedida por un proceso de elección por voto universal, es decir, el presidente designaría a los prefectos, como señalaba la Constitución y tal como se hacía antes, pero sólo a quienes resultaran ganadores de los comicios ${ }^{5}$ y 2) una ley especial que modificó el Código Electoral para que pudiera ser el presidente del país quien convoque a dichas elecciones. ${ }^{6}$ Finalmente, éstas se realizaron el 18 de diciembre de 2005, de forma simultánea a la elección presidencial, pero con la diferencia de que los candidatos ganadores sólo necesitaban mayoría relativa de votos.

El complejo proceso para llevar a cabo las elecciones de prefectos muestra que existían varias trabas legales que no era fácil sortear a efecto de que los comicios no fueran impugnados legalmente. Sin embargo, las mismas fueron superadas por tres presidentes distintos y por el Congreso, compuesto en su mayoría por partidos tradicionales que luego desaparecieron de la escena política nacional. Ello evidencia que había plena voluntad política para una reforma histórica que rompía con un pilar del Estado unitario boliviano y que, sin duda, debilitaba el poder de los futuros presidentes, quienes sólo podrían nombrar a algún colaborador suyo en caso de que alguno de los prefectos electos sea apartado de sus funciones.

¿Fue incluyente o excluyente la reforma que impulsó la elección de prefectos por voto universal? Puesto que a través de comicios prefecturales los presidentes enfrentan la posibilidad de que opositores suyos accedan a los gobiernos departamentales, algo que antes no era posible, resulta muy claro que esta reforma fue incluyente. Con ella los partidos buscaban minimizar pérdidas futuras ante un eventual desplazamiento de posiciones de poder a nivel nacional; es decir, la reforma les abría la posibilidad de replegarse al

\footnotetext{
${ }^{5}$ Ley Interpretativa del Articulo 109 de la Constitución Política del Estado, de 6 de julio de 2005.

${ }^{6}$ Se trata de la Ley Especial, complementaria al Código Electoral, a la Ley de Partidos Políticos y a la Ley de Agrupaciones Ciudadanas y Pueblos Indígenas, para la Elección y Selección de Prefectos(as) de Departamento, de 8 de abril de 2005.
} 
ámbito departamental. Además, no se requería una fuerza electoral muy grande para ello, dado que bastaba una mayoría simple de votos para que un candidato sea electo como prefecto y, en último caso, era posible incluso que algún político decida postular por medio de alguna agrupación ciudadana. En ese sentido, el cambio institucional descrito confirma el planteamiento de Buquet y una parte de la afirmación del último párrafo del apartado 2: la reforma que permitió que los prefectos sean electos fue impulsada por una coalición política declinante que, debido a problemas de legitimidad, vislumbraba una futura derrota y buscaba minimizar sus pérdidas.

\section{Nueva Constitución: ¿nuevas reglas para elegir prefectos?}

La Nueva Constitución Política del Estado, aprobada en referéndum nacional en enero de $2009,{ }^{7}$ establece que los gobernadores de departamento (antes llamados prefectos) deben ser electos mediante sufragio universal, lo que da continuidad a las reformas impulsadas en 2005, que posibilitaron por primera vez que la población boliviana elija a dichas autoridades. Además, señala que su mandato es de cinco años y que pueden ser reelectos de manera continua por una sola vez, al igual que el presidente. Por último, dicha norma determina que solamente se puede convocar a elecciones anticipadas de gobernador departamental cuando ocurre su renuncia, muerte o revocatoria durante la primera mitad del mandato.

Si consideramos que la convocatoria a la Asamblea Constituyente que redactó la Constitución (aprobada luego en referéndum) fue parte sustancial del programa electoral con que el Movimiento al Socialismo ganó la elección presidencial de 2005, y que lo que establece sobre la elección de gobernadores reduce el poder que al respecto tenía el presidente bajo la Constitución anterior, de manera tal que posibilita que las fuerzas opositoras accedan a los espacios de poder locales que buscaron con la reforma de 2005, resulta natural preguntarse por qué el MAS no buscó la manera de que la nueva norma

\footnotetext{
7 La Constitución fue aprobada con el 61,4 por ciento de votos a favor. En dicho referéndum nuevamente fue relevante la identificación indígena, ya que, de acuerdo a datos de LAPOP, el 93 por ciento de indígenas ( 86 por ciento de quechuas y 96 por ciento de aymaras) manifestó haber votado a favor del cambio constitucional, en comparación con el 77 por ciento de mestizos y el 58 por ciento de blancos. Estos datos muestran que el apoyo indígena es fundamental para el MAS.
} 
favorezca el control político del gobierno central en los departamentos. Esta interrogante es incluso más relevante si tomamos en cuenta que los resultados de las elecciones prefecturales de 2005 le fueron altamente desfavorables (sólo ganó en tres de los nueve departamentos), y que entre ese año y 2009 la tensión con los prefectos opositores fue de tal magnitud que sólo pudo resolverse cuando todos ellos y el presidente se sometieron a un referéndum revocatorio. $^{8}$

Si bien el Movimiento al Socialismo fue el actor más visible que proponía la realización de una Asamblea Constituyente dirigida a refundar el país, esta demanda había ingresado a la agenda política desde mucho antes. Hasta donde he podido rastrear fue Max Fernández, líder de Unidad Cívica Solidaridad (UCS), quien primero propuso la realización de una Asamblea Constituyente en la campaña presidencial de 1993 con el objetivo de construir una democracia participativa. En las elecciones de 2002 este planteamiento fue más extendido debido al desgaste del sistema político y estuvo presente en los programas de gobierno del MAS, de Nueva Fuerza Republicana (NFR) e incluso del Movimiento de Izquierda Revolucionaria (MIR), que era uno de los partidos que había co-gobernado el país en varias ocasiones. Para las elecciones anticipadas de 2005, y en plena crisis de legitimidad del sistema político y de los partidos, prácticamente todos los candidatos presidenciales propusieron instalar una Asamblea Constituyente para enfrentar los profundos problemas de representación existentes (sin duda que unos lo hacían por convicción y otros sólo por estrategia)..$^{9}$ En ese sentido, la modificación de la Constitución Política a través de delegados expresamente elegidos para ello mediante voto popular se convirtió en una agenda nacional que ningún gobierno hubiera podido ignorar.

Otro tema que había estado en la agenda desde antes, e incluso por más tiempo, era la descentralización administrativa. Desde mediados de los ochenta los principales partidos políticos, y con independencia de su signo ideológico, plantearon en todas las campañas electorales la necesidad de descentralizar el país, aunque ninguno dio mayores detalles sobre esa propuesta. Además, los departamentos con economías más dinámicas (en especial los productores de petróleo) usualmente demandaban avanzar hacia

\footnotetext{
8 Un análisis de esos eventos se encuentra en Torrico (2016).

9 Para más detalles véase el trabajo de H.C.F. Mansilla y Carlos Toranzo citado en la bibliografía y también los programas de gobierno del MAS (2002 y 2005), MIR (2002), NFR (2002), UCS (2002), MNR (2005), PODEMOS (2005) y UN (2005).
} 
la descentralización con el fin de aumentar su participación en los recursos que ellos mismos generaban, ya que éstos eran distribuidos por el gobierno central.

Aunque ciertamente el proceso de descentralización administrativa avanzó desde la década del noventa y se incrementaron los recursos que manejaban los departamentos y municipios, la demanda local por una profundización del mismo se mantuvo y los partidos no dejaron de ofertar una respuesta positiva. Sin embargo, nunca se habló de la posibilidad de que los prefectos de departamento dejen de ser designados por el presidente y sean electos. Esa propuesta emergió durante la crisis política que se inició en 2003 y fue expresada con fuerza en enero de 2005 , en un cabildo realizado en la ciudad de Santa Cruz que reunió a más 100 mil personas y exigió la instauración de un gobierno autonómico. La propuesta salió del Comité Cívico de esa ciudad, el cual también había convocado al mencionado cabildo.

Tradicionalmente el Comité Cívico de Santa Cruz ha tenido estrechos lazos con la clase empresarial cruceña y con los partidos políticos dominantes del periodo 1985-2005..$^{10}$ En el momento del cabildo sus líderes era Rubén Costas y Carlos Dabdoub, el primero se convirtió luego en gobernador electo de ese departamento (ganando tres elecciones consecutivas y siendo una de las figuras más visibles de la oposición al gobierno de Evo Morales) y el segundo había sido previamente diputado y Ministro de Salud en el gobierno de Jaime Paz Zamora (respaldado por la coalición MIR-ADN), y posteriormente fue candidato a la vicepresidencia por Unidad Nacional, que a la fecha es el partido opositor más importante en Bolivia y que tiene sus orígenes en el MIR. La poca capacidad de negociación que en ese momento tenía el presidente Carlos Mesa (su candidatura a la vicepresidencia se había debido a su buena imagen como periodista y escritor, la que explotó con éxito Sánchez de Lozada), hizo que la demanda de los dirigentes cruceños le sea fácilmente impuesta y que la mayoría del Congreso la apoye aprobando las normas que eran necesarias para que la elección de prefectos pueda ser posible.

Un indicador de que la reforma política que posibilitó que los prefectos sean electos era parte de un cálculo estratégico de partidos políticos en declive con el fin de preservar espacios de poder a costa de debilitar al gobierno central, fue que la aprobación del Decreto Supremo 27988 generó una inmediata reacción adversa de Evo Morales, quien convocó a realizar

\footnotetext{
10 Para más detalles véase el trabajo de Freddy Gonzáles citado en la bibliografía.
} 
movilizaciones populares en contra del llamado a elecciones para prefectos. Para Morales, quien en ese momento era el candidato con más posibilidades de ganar la presidencia, la reforma implicaba una inadmisible cesión de poder por parte del gobierno central. ${ }^{11}$ En los hechos, Evo Morales estaba defendiendo una de las atribuciones más importantes que como presidente aspiraba a tener en poco tiempo, pero finalmente se impuso la mayoría en el Congreso, conformada por la coalición declinante. Ése fue el momento de ruptura entre Morales y Mesa, ${ }^{12}$ quien pocos meses después se vio obligado a renunciar a la presidencia.

Si bien Morales no podía eludir la realización de una Asamblea Constituyente, sí tenía margen de maniobra para encarar la demanda por mayor descentralización; después de todo los detalles de la misma nunca fueron precisos. Es así que la propuesta de descentralización del MAS durante la campaña electoral de 2005 fue expuesta en términos muy vagos.

El Estado deberá jugar jugar un papel central en el proceso de descentralización política, económica y social, compatibilizando estrategias de desarrollo nacional y regional para cumplir con los objetivos de desarrollo económico y social. [...] Así, el proceso de descentralización debe asumir la responsabilidad de reducir las asimetrías regionales, eliminar la exclusión económica, política, social y cultural de la mayoría de la población, asimismo deberá asegurar la satisfacción de las necesidades básicas de la mayoría de la población en alimentación y nutrición, salud, educación, vivienda y saneamiento básico, energía y comunicación. (MAS 2005, 20)

¿Por qué el MAS no anuló la elección de prefectos en la Nueva Constitución y le devolvió al presidente la atribución de nombrarlos? Durante el desarrollo de la Asamblea Constituyente se vivieron muchos momentos de alta tensión. Tan sólo la discusión del reglamento de debates duró más de seis meses, y la inclusión en el temario de un punto referido a si la Constitución debía definir a la capital y sede de los poderes del país amenazó seriamente con paralizar e incluso abortar todo el proceso. Si bien el partido gobernante contaba con mayoría de asambleístas, carecía de la mayoría de dos tercios requerida para aprobar su propuesta. El enfrentamiento escaló a tal nivel que la Constituyente tuvo que ser trasladada a otra ciudad y aprobada en un cuartel militar. ${ }^{13}$ De haberse intentado dar marcha atrás en la elección de prefectos el

\footnotetext{
11 Sobre este episodio, véase Pascual Albanese, "Bolivia es una bomba de tiempo" (2005).

12 Hasta ese momento Evo Morales había tenido una actitud ambigua respecto al gobierno de Carlos Mesa, algunas veces de apoyo y otras de crítica.

13 Para más detalles véase Grace Ivana Deheza (2007 y 2008) y Fredrik Uggla (2009).
} 
conflicto habría sido mayor y posiblemente se habría propagado a otros departamentos. Es probable que ése haya sido un aspecto que el MAS tomó en cuenta para no presentar una propuesta en ese sentido. Aunque esto último no deja de ser una conjetura, lo cierto es que no hay evidencia de que el oficialismo se haya planteado retornar al estatus quo anterior en relación a la designación de los prefectos.

Anteriormente se mostró que luego de la elección de Evo Morales como presidente mejoró la satisfacción con la democracia y la imagen de los partidos en la población. A partir de 2006 se observa también un aumento de la confianza en el presidente y en la gestión de las prefecturas, las cuales ya estaban encabezadas por prefectos electos. Incluso en 2008, año en que la Asamblea Constituyente finalizó y aprobó el proyecto de Constitución que debía refrendarse mediante un referéndum, ambos niveles de confianza fueron similares. (Fig. 4) Ese contexto de apoyo creciente a las autoridades departamentales electas hacía inviable cualquier intención de retomar el control de las prefecturas por parte del gobierno central a través del nombramiento discrecional de prefectos, como ocurría antes.

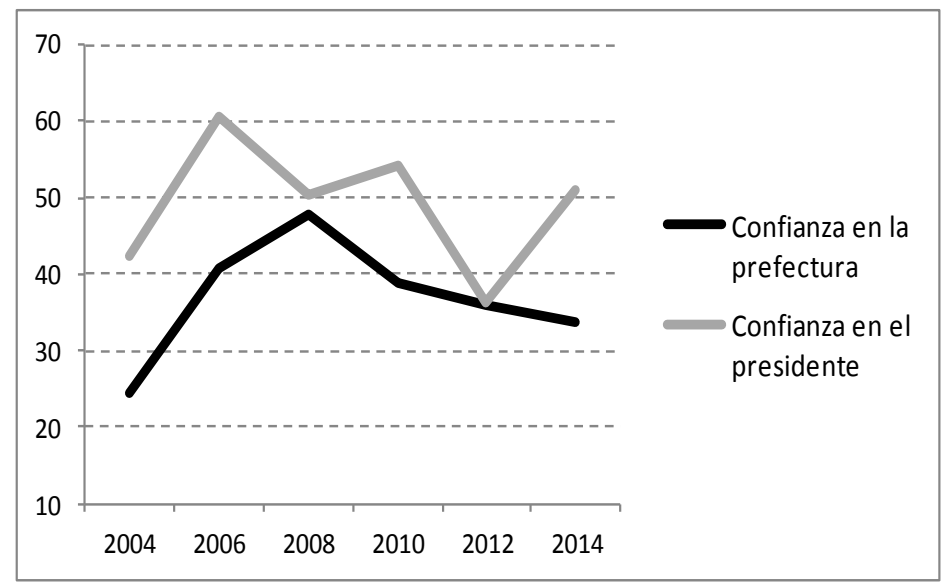

Figura 4: Porcentaje de personas que confían en el presidente y en la prefectura Fuente: elaboración propia con datos de LAPOP 
La Nueva Constitución Política no alteró la elección por voto universal para elegir a los prefectos que se había establecido en 2005, poco antes del primer triunfo electoral de Evo Morales, pero realizó algunos ajustes que le permitían al MAS aumentar sus posibilidades futuras de triunfo en los departamentos, a saber: 1) en 2005 bastaba con una mayoría simple para ser electo como prefecto, pero en la Nueva Constitución se estableció la fórmula de mayoría absoluta con segunda vuelta; y 2) antes no se contemplaba la posibilidad de reelección para ninguna autoridad ejecutiva, pero a partir de 2009 tanto los prefectos como el presidente pueden ser reelectos de forma inmediata. Buquet señala que ese tipo de cambios son típicos de coaliciones ascendentes que buscan diseñar reglas excluyentes para poder gobernar. Así, las candidaturas opositoras requieren incremento de votos para imponerse electoralmente (lo que disminuye sus posibilidades de triunfo frente a la maquinaria oficialista o a la popularidad de su líder) y los gobernadores electos pueden permanecer mucho tiempo en el cargo. En el caso de los gobernadores del Movimiento al Socialismo, además, habría el impulso adicional de la popularidad de Evo Morales.

Al incluir en la Constitución reglas que mejoren sus posibilidades de triunfo en elecciones para gobernadores, el MAS optó por una estrategia que le dé resultados positivos sin tener que alterar radicalmente la reforma de 2005. En sentido estricto, no estableció una manera distinta a la de 2005 para designar prefectos (lo que respalda la afirmación realizada al final del apartado 2 de que el nuevo actor dominante no tuvo posibilidad de eliminarla), pero aprobó cambios de menor nivel con la intención de aumentar su control político a nivel subnacional. ¿Dio resultado?

\section{Competencia política en las gobernaciones departa- mentales}

Las elecciones para elegir prefectos introdujeron una dinámica política inexistente previamente en Bolivia. Por primera vez era posible que el ámbito nacional no sea el único espacio importante de competencia política. Para analizar esta coyuntura es posible recurrir a múltiples indicadores, y en este caso emplearemos el "número efectivo de partidos" y la "polarización de votos". El primero contabiliza los partidos participantes en una elección y a la vez los pondera por su fuerza relativa, que es el porcentaje de votos que obtiene. "Polarización" describe cuán igualada fue la repartición de votos; así, cuando hay dos partidos y cada uno obtiene 50 por ciento de los sufragios, el 
indicador adquiere su valor máximo que es uno; en cambio, cuando todos los votos van a un solo partido el valor es de cero. La relación entre ambos indicadores es curvilínea, es decir, cuando el "número efectivo" es reducido o elevado, la "polarización" es baja, y cuando los valores del anterior son intermedios el último indicador es alto. ${ }^{14}$ Empleando dichos indicadores, a continuación se analizará la competencia electoral en elecciones presidenciales y prefecturales a partir de 2005.

Como se observa en el siguiente gráfico (Fig. 5), hay más competencia en elecciones para prefectos o gobernadores que en las presidenciales. En todos los años el "número efectivo" y la "polarización" son mayores en las contiendas departamentales que en la nacional, lo que significa que no sólo hay más candidatos compitiendo a nivel subnacional, sino que también esas elecciones son más reñidas. Esto muestra que el dominio político a nivel nacional que el MAS ostenta desde 2005 y que tanta atención ha despertado a nivel regional, se reduce cuando analizamos los resultados a nivel subnacional:

\begin{tabular}{|l|c|c|c|c|}
\hline $\begin{array}{c}\text { Tipo de } \\
\text { elección }\end{array}$ & $\begin{array}{c}\text { Año de } \\
\text { elecciones }\end{array}$ & $\begin{array}{c}\text { Núm. Partidos } \\
\text { participantes }\end{array}$ & $\begin{array}{c}\text { Núm. Efectivo } \\
\text { de Partidos }\end{array}$ & $\begin{array}{c}\text { Polarización } \\
\text { en votos }\end{array}$ \\
\hline \multirow{3}{*}{ Presidencial } & 2005 & 8 & 2,62 & 0,808 \\
\cline { 2 - 5 } & 2009 & 8 & 2,06 & 0,811 \\
\cline { 2 - 5 } & 2014 & 5 & 2,26 & 0,797 \\
\hline \multirow{2}{*}{$\begin{array}{l}\text { Prefectos o } \\
\text { Gobernadores* }\end{array}$} & 2005 & 5 & 2,93 & 0,826 \\
\cline { 2 - 5 } & 2010 & 4,56 & 2,38 & 0,854 \\
\cline { 2 - 5 } & 2015 & 5,11 & 2,55 & 0,823 \\
\hline
\end{tabular}

Figura 5: Elecciones presidenciales y prefecturales: competencia electoral comparada

Fuente: elaboración propia

*Los indicadores refieren el promedio de todos los departamentos

\footnotetext{
${ }^{14}$ La fórmula de "Número efectivo de partidos" es la siguiente: $N=1 / \sum p_{i}{ }^{2}$, donde $p_{i}{ }^{2}$ es el cuadrado de la proporción de votos recibida por cada partido. La fórmula de "Polarización" es la siguiente: $P=\Sigma p_{i}\left(p_{i}\left(1-p_{i}\right)\right.$, donde $p_{i}$ es la proporción de votos obtenida por cada partido. Al respecto, véase los trabajos de Markku Laakso y Rein Taagepera (1979) y de José Montalvo y Martha Reynal-Querol (2005) consignados en la bibliografía.
} 
Un rasgo novedoso que introdujo la elección de prefectos o gobernadores fue que surgió la posibilidad de registrar partidos o agrupaciones ciudadanas de alcance departamental, lo que reduce los costos de organización para presentar candidaturas subnacionales (recordemos que antes sólo existían en Bolivia organizaciones políticas nacionales) y hace posible que la competencia política sea amplia y diversa. Es así que en 2005, 2010 y 2015 sólo el Movimiento al Socialismo ha presentado candidatos en todos los departamentos, y que en esas tres elecciones ha habido en promedio 15 partidos y agrupaciones que no han presentado candidatura en más de un departamento. Ello sin duda refleja que el MAS es el único partido de alcance verdaderamente nacional y que la oposición está muy fragmentada (algo que no es muy saludable para la democracia), pero también muestra un panorama político subnacional muy dinámico y competitivo, que contrasta con lo que sucede en elecciones presidenciales.

La competencia política dinámica que existe a nivel subnacional no implica necesariamente que el conflicto se atenúe. Como se señaló antes, las elecciones prefecturales de 2005 fueron adversas para el MAS a pesar de que se efectuaron simultáneamente a la elección presidencial. Eso abrió un periodo de hostilidad entre el gobierno central y los prefectos de oposición que llevó a la realización de un referéndum revocatorio en agosto 2008, del cual Evo Morales salió fortalecido y la oposición muy debilitada, ya que dos de las autoridades departamentales fueron revocadas. Poco tiempo después, en un episodio de enfrentamientos entre simpatizantes del oficialismo y de la oposición en Pando, que arrojó el saldo de 20 personas muertas (la mayoría campesinos), el prefecto de ese departamento fue apresado y destituido del cargo por el gobierno central. ${ }^{15}$ Las acefalías en esos tres casos fueron cubiertas por nombramientos directos del presidente, ya que así lo establecía el Decreto Supremo 27988 que había convocado a elecciones prefecturales en 2005. En este caso, la regla diseñada por la coalición declinante terminó beneficiando al Movimiento al Socialismo, lo que evidencia que el efecto final del cambio institucional no podía ser totalmente anticipado, es decir, que la incertidumbre no puede eliminarse.

\footnotetext{
${ }^{15}$ Este suceso fue investigado por una Comisión de la Unión de Naciones Suramericanas (UNASUR), concluyendo que "los agresores de los campesinos lo hicieron de forma organizada y respondían según algunos testimonios a una cadena de mando y contaban con funcionarios y bienes del gobierno departamental". Además, recomendó que el asesinato con arma de fuego de dos funcionarios de la Prefectura de Pando fuera investigada por las autoridades de justicia. Esto evidencia que el conflicto entre el gobierno y algunas oposiciones departamentales alcanzó altos niveles de violencia.
} 
Los conflictos entre el MAS y las oposiciones departamentales continuaron después de la reelección de Evo Morales en 2009, cuando éste consolidó su liderazgo y su partido obtuvo mayoría calificada en ambas cámaras del Congreso. Así, a los prefectos de los departamentos de Beni y Tarija (ambos opositores) se les inició procesos judiciales bajo la acusación de corrupción, y en virtud de la Ley Marco de Autonomías y Descentralización № 031/2010, aprobada en el segundo gobierno de Morales y que establecía la remoción de autoridades con la sola acusación formal, ambos fueron destituidos. ${ }^{16}$ El siguiente cuadro muestra los resultados de elecciones para prefectos o gobernadores, detallando si los ganadores pertenecían al MAS o a alguna candidatura de oposición y si lograron finalizar su periodo de gobierno. Con ello se da cuenta del conflicto entre el gobierno y las oposiciones departamentales.

\begin{tabular}{|l|c|c|c|c|c|c|}
\hline Departamento & $\mathbf{2 0 0 5}$ & $\begin{array}{c}\text { ¿Finalizó } \\
\text { efodo? }\end{array}$ & $\mathbf{2 0 1 0}$ & $\begin{array}{c}\text { ¿Finalizó } \\
\text { el } \\
\text { periodo? }\end{array}$ & $\mathbf{2 0 1 5}$ & $\begin{array}{c}\text { ¿Aún en } \\
\text { funciones? }\end{array}$ \\
\hline Chuquisaca & MAS & No & MAS & Sí & MAS & Sí \\
\hline La Paz & Oposición & No & MAS & Sí & Oposición & Sí \\
\hline Cochabamba & Oposición & No & MAS & Sí & MAS & Sí \\
\hline Oruro & MAS & Sí & MAS & Sí & MAS & Sí \\
\hline Potosí & MAS & Sí & MAS & Sí & MAS & Sí \\
\hline Tarija & Oposición & Sí & Oposición & No & Oposición & Sí \\
\hline Santa Cruz & Oposición & Sí & Oposición & Sí & Oposición & Sí \\
\hline Beni & Oposición & Sí & Oposición & No & MAS & Sí \\
\hline Pando & Oposición & No & MAS & Sí & MAS & Sí \\
\hline
\end{tabular}

Figura 6: Candidaturas ganadoras en elecciones de prefectos/gobernadores Fuente: elaboración propia

\footnotetext{
16 Los artículos de la mencionada Ley que permitían ese tipo de remoción fueron declarados inconstitucionales por el Tribunal Constitucional Plurinacional en 2013, pero dichas autoridades no fueron repuestas en el cargo. Los procesos judiciales tampoco avanzaron y a la fecha ni siquiera se ha concluido la etapa de acusación.
} 
Puede observarse que el éxito electoral del MAS en elecciones departamentales ha ido creciendo en el tiempo. En 2005 sólo había ganado en tres departamentos, pero en 2010 y 2015 su triunfo llegó a seis. A ello contribuyeron dos factores: 1) los ajustes de doble vuelta y reelección inmediata que incluyó la Nueva Constitución, y 2) los conflictos con la oposición, que se resolvieron a su favor en virtud de reglas elaboradas previamente por ésta (cuando era una coalición declinante) como de nuevas reglas diseñadas con el objetivo de controlar o sancionar principalmente a las autoridades opositoras. En relación al primer factor se debe mencionar que aunque el único prefecto que ha sido reelecto dos veces desde 2005 pertenece a la oposición (Rubén Costas de Santa Cruz), esa figura ha beneficiado en mayor medida al MAS, que ha logrado reelegir a sus gobernadores en Chuquisaca y en Pando (además de que las dos reelecciones de Evo Morales han consolidado el dominio de su partido a nivel nacional). Sobre el segundo factor se puede ver que cinco de los seis prefectos electos que no han concluido su periodo de gobierno son de la oposición; que en la elección inmediata posterior en cuatro de esos cinco departamentos el MAS logró el triunfo, y que ningún gobernador de dicho partido ha sido destituido por la aplicación de la Ley 031/2010.

Lo anterior refleja que, aunque a nivel subnacional hay mayor competencia electoral que a nivel nacional, el Movimiento al Socialismo se ha beneficiado de reglas institucionales anteriores a su consolidación como partido dominante a nivel nacional, como también de nuevas reglas dirigidas a disminuir el margen de acción de las oposiciones locales y, eventualmente, a posibilitar el aumento de espacios de poder en los departamentos. Esto confirma el marco teórico propuesto en este artículo para explicar el cambio institucional en las reglas que afectan la competencia política: las coaliciones políticas declinantes llevan a cabo reformas incluyentes con el fin de minimizar pérdidas futuras que no pueden ser revertidas por las coaliciones ascendentes que luego llegan al poder. Ahora bien, esta explicación debe ser complementada, a partir de los hallazgos de este estudio, señalando que las coaliciones ascendentes intentan reducir el grado de inclusión de las reglas a través de cambios normativos de menor nivel que disminuyen la competencia política.

De cualquier manera, la afirmación del apartado 2 puesta a prueba se confirma en su mayor parte por el análisis precedente, ya que el MAS no tuvo posibilidad de eliminar la elección popular de prefectos o gobernadores, lo que tuvo como efecto que en Bolivia haya mayor competencia política a nivel subnacional de la que existe a nivel nacional y que haya mayor pluralidad en los gobiernos departamentales de la que había bajo la regla anterior en que el 
presidente nombraba a esas autoridades. Es decir, a pesar de que el Movimiento al Socialismo ha aumentado notablemente su éxito en elecciones departamentales, los partidos y grupos de oposición conservan espacios que no tendrían bajo la regla de designación anterior.

\section{Conclusiones}

A lo largo de este artículo se ha analizado el origen y los efectos del cambio en las reglas que afectan a la competencia política. Para ello se ha propuesto un marco teórico explicativo que ha sido contrastado con el estudio de la reforma política de 2005 que permitió que los prefectos (ahora llamados gobernadores) sean electos por voto popular en Bolivia. El análisis ha confirmado la validez de la explicación propuesta, pero también ha revelado que la misma requiere ser complementada. Allí radica el aporte de este trabajo a la literatura sobre cambio institucional, ya que la teoría que se contrastó fue construida en base a una revisión de evidencia. Si bien los estudios previos nos señalaban que los cambios son impulsados por coaliciones declinantes (o ascendentes) que anticipan futuras derrotas (o victorias) electorales, en función de lo cual diseñan reglas incluyentes (o excluyentes) que las beneficien y que difícilmente pueden ser modificadas, el análisis del caso nos mostró que las nuevas coaliciones no necesitan dar marcha atrás con esos cambios para reducir su efecto, ya que pueden impulsar reformas de menor nivel o desplegar estrategias políticas extra institucionales que les beneficiarán. Sin embargo, aunque el efecto del cambio institucional puede ser disminuido por las nuevas coaliciones, no consigue ser eliminado, con lo que quienes las impulsaron ven que sus objetivos se cumplen en alguna medida.

En Bolivia el cambio institucional consistió en que el prefecto o gobernador de cada departamento ya no es directamente nombrado por el presidente, sino que es electo. Ello disminuyó las atribuciones que al presidente le permitían tener control político a nivel subnacional y permitió que grupos y personas cercanos o pertenecientes a los partidos que habían dominado la política entre 1985 y 2005 pudiesen tener espacios de poder en un contexto totalmente adverso, en que el propio sistema de partidos desapareció y un partido muy poderoso emergió con dominio absoluto a nivel nacional.

El Movimiento al Socialismo, que actualmente es dominante en Bolivia, no pudo o no se planteó revertir la reforma política de 2005 y la incorporó a la Constitución aprobada en 2009. Sin embargo, tampoco se planteó asumir del 
todo la incertidumbre de la nueva regla y se enfocó en impulsar cambios de menor nivel (mayoría absoluta en lugar de mayoría relativa para elegir a prefectos o gobernadores, posibilidad de reelección para ellos y para el presidente, aprobación de normas que permiten la remoción de autoridades y que fueron aplicadas selectivamente) para recuperar en alguna medida el control político perdido. Con ello logró mejorar su éxito electoral en elecciones departamentales, pero la oposición pudo conservar algunos espacios a los que no habría podido acceder de no haberse implementado la reforma. El saldo final es que a nivel subnacional hay mayor competencia política que a nivel nacional.

Este artículo confirma la importancia de los estudios de caso para el avance teórico, en especial cuando se quiere explicar fenómenos políticos en contextos en que no hay garantía sobre el cumplimiento estricto de las reglas, como ocurre en prácticamente toda América Latina. También confirma que los actores pueden transformar las reglas para beneficiarse en el futuro y que una vez que éstas son puestas en funcionamiento es muy difícil eliminarlas.

\section{Bibliografia citada}

ALBANESE, Pascual. 2005. "Bolivia es una bomba de tiempo".

[http://www.harrymagazine.com/200501/bombadetiempo.htm] página descargada el 10 de noviembre, 2016.

BUQUET, Daniel. 2007. "Entre la legitimidad y la eficacia: Reformas en los sistemas de elección presidencial en América Latina". Revista Uruguaya de Ciencia Política 16 (1): 35-49.

CONSTITUCIÓN POLÍTICA DE LA REPÚBLICA DE BOLIVIA. 1967. Con reformas introducidas por la Ley № 1585 del 12 de agosto de 1994, texto concordado de 1995 sancionado por Ley № 1615 del 6 de febrero de 1995, reformas introducidas por Ley № 2410 del 8 de agosto de 2002, reformas introducidas por Ley № 2631 del 20 de febrero de 2004, y reformas introducidas por Ley № 3089 del 6 de julio de 2005.

[http://pdba.georgetown.edu/Constitutions/Bolivia/consboliv2005.html] página descargada el 15 de octubre 2016.

COX, Gary. 1997. Making votes count. Strategic Coordination in the World's Electoral Systems. Nueva York: Cambridge University Press. 
DECRETO SUPREMO 27988. 28 de enero 2005.

[http://200.87.110.166/sealinea/pdf/01/001.pdf] página descargada el $10 \mathrm{de}$ noviembre 2016.

DECRETO SUPREMO 28077. 8 de abril 2005.

[http://200.87.110.166/sealinea/pdf/05/004.pdf] página descargada el $10 \mathrm{de}$ noviembre 2016.

DECRETO SUPREMO 28229. 6 de julio 2005.

[http://www.lexivox.org/norms/BO-DS-28229.xhtml] página descargada el 10 de noviembre 2016.

DEHEZA, Grace Ivana. 2007. "Bolivia 2006: Reforma estatal y construcción del poder". Revista de Ciencia Política 27. Número especial. 43-57.

---. 2008. "Bolivia: ¿Es posible la construcción de un nuevo Estado? La Asamblea Constituyente y las Autonomías departamentales". Revista de Ciencia Política 28 (1): 61-79.

DO ALTO, Hervé. 2011. “Un partido campesino en el poder: una mirada sociológica del MAS Boliviano". Nueva sociedad 234. Julio-agosto. 95-111.

DUVERGER, Maurice. 1957. Los partidos políticos. México: FCE.

GARCÍA LINERA, Álvaro. 2006. "El evismo: lo nacional-popular en acción”. OSAL. Observatorio Social de América Latina VI (19): 25-32.

GONZÁLEZ, Freddy. 2003. Comité Cívico pro-Santa Cruz: grupos de poder y liderazgo regional. Análisis sociopolítico y cultural 1980-2000. La Paz: PIEB.

JUNG, Jai Kwan y Christopher J. Deering. 2015. “Constitutional Choices:

Uncertainty and Institutional Design in Democratising Nations". International Political Science Review 36 (1): 60-77.

[http://ips.sagepub.com/content/36/1/60.full.pdf+html] página descargada el 10 de agosto 2016.

LAAKSO, Markku y Rein Taagepera. 1979. "Effective Number of Parties: A Measure with Application to West Europe”. Comparative Political Studies 12 (1): 3-27. [http://cps.sagepub.com/content/12/1/3.full.pdf+html] página descargada el 10 de agosto 2016.

LAPOP (Latin American Public Opinion Project) [www.lapopsurveys.org] y [http://www.vanderbilt.edu/lapop-espanol/datos-encuestas.php] páginas descargadas el 10 de noviembre 2016.

LATINOBARÓMETRO. Opinión Pública Latinoamericana. 1995-2006. Banco de Datos. [http://www.latinobarometro.org/latContents.jsp] 
LEY N²771 DE AGRUPACIONES CIUDADANAS Y PUEBLOS INDÍGENAS. 7 de julio de 2004.

http://aclo.org.bo/electoral/images/stories/leyes/normativaslegales/LeyAgr upacionesPueblos.pdf] página descargada el 15 de octubre 2016.

LEY N³015. LEY ESPECIAL, COMPLEMENTARIA AL CÓDIGO ELECTORAL, A LA LEY DE PARTIDOS POLÍTICOS Y A LA LEY DE AGRUPACIONES CIUDADANAS Y PUEBLOS INDÍGENAS, PARA LA ELECCIÓN Y SELECCIÓN DE PREFECTOS(AS) DE DEPARTAMENTO. 8 de abril 2005.

[http://www.gacetaoficialdebolivia.gob.bo/index.php/normas/descargar/103 03] página descargada el 15 de octubre 2016.

LEY 3090 INTERPRETATIVA DEL ARTÍCULO 109 DE LA CONSTITUCIÓN POLÍTICA DEL ESTADO. 6 de julio de 2005. [http://www.lexivox.org/norms/BO-L3090.xhtml] página descargada el 10 de agosto 2016.

LEVITSKY, Steven y María Victoria Murillo. 2009. "Variation in Institutional Strength". Annual Review of Political Science 12. 115-33.

[http://www.annualreviews.org/doi/pdf/10.1146/annurev.polisci.11.091106. 121756] página descargada el 10 de agosto 2016.

LOZADA PEREIRA, Blithz y Marco Antonio Saavedra Mogro. 1998. Democracia, pactos y élites. Genealogía de la gobernabilidad en el neoliberalismo. La Paz: Instituto de Investigaciones de Ciencia Política, UMSA.

MANSILLA, H.C.F. y Carlos Toranzo, eds. 1993. Foro político electoral. Los programas de los partidos. La Paz: CEBEM-ILDIS.

MAS (Movimiento al Socialismo). 2002. "Lineamientos generales del programa de gobierno”. Opiniones y Análisis 56. Elecciones Generales 2002-2007. Propuestas electorales. La Paz: Fundemos. 59-88.

---. 2005. Programa de Gobierno 2006-2010. Bolivia Digna, Soberana y Productiva para Vivir Bien.

[http://www.oep.org.bo/proces_electoral/generales2005/comunicacion/egp 2005_programa_mas.pdf] página descargada el 10 de noviembre 2016.

McFAUL, Michael. 1999. "Institutional Design, Uncertainty, and Path Dependency during Transitions: Cases from Russia". Constitutional Political Economy 10 (1): 27-52.

MIR (Movimiento de Izquierda Revolucionaria). 2002. "Plan trabajo Bolivia para los Bolivianos. Programa de gobierno 2002/2007”. Opiniones y Análisis 56. Elecciones Generales 2002-2007. Propuestas electorales. La Paz: Fundemos. 135-202. 
MNR (Movimiento Nacionalista Revolucionario). 2005. Un Programa para el Cambio: Participación Productiva.

[http://www.oep.org.bo/proces_electoral/generales2005/comunicacion/egp 2005_programa_mnr.pdf] página descargada el 10 de noviembre 2016.

MONTALVO, José G. y Marta Reynal-Querol. 2005. “Ethnic Polarization, Potential Conflict and Civil Wars". The American Economic Review 95 (3): 796-816.

NFR (Nueva Fuerza Republicana). 2002. Plan de Gobierno 2002-2007. La Paz, mimeo.

NORTH, Douglass C. 1993. Instituciones, cambio institucional y desempeño económico. México: FCE.

NUEVA CONSTITUCIÓN POLÍTICA DEL ESTADO PLURINACIONAL DE BOLIVIA. 2009. Vicepresidencia del Estado Plurinacional de Bolivia.

[http://www.vicepresidencia.gob.bo/IMG/pdf/ncpe_cepd.pdf] página descargada el 10 de noviembre 2016.

PODEMOS (Poder Democrático Social). 2005. Programa de gobierno 2006-2010. [http://www.oep.org.bo/proces_electoral/generales2005/comunicacion/egp 2005_programa_podemos.pdf] página descargada el 10 de noviembre 2016.

SVAMPA, Maristella. 2010. "Movimientos Sociales, matrices socio-políticos y nuevos escenarios en América Latina". Working Papers 1. OneWorld Perspectives.

[https://kobra.bibliothek.uni-kassel.de/bitstream/urn:nbn:de:hebis:342010110334865/1/OWP_Working_Paper_2010_01.pdfl página descargada el 5 de noviembre 2016.

TORRICO, Mario. 2014. "Bolivia: nuevo sistema electoral presidencial y coordinación Política de los partidos". Perfiles Latinoamericanos 22 (43): 77102.

---. 2016. "Una década de Evo Morales en el poder: Lecciones pasadas y necesidades futuras de la democracia boliviana". Foreign Affairs Latinoamérica 16 (3): 36-46.

TSEBELIS, George. 1990. Nested Games: Rational Choice in Comparative Politics. Los Ángeles: University of California Press. [http://www.scielo.org.mx/pdf/perlat/v22n43/v22n43a4.pdf] página descargada el 10 de noviembre 2016.

UCS (Unidad Cívica Solidaridad). 2002. "Pacto con Bolivia. Programa de gobierno 2002-2007". La Paz: Opiniones y Análisis 56. Elecciones Generales 2002-2007. Propuestas electorales. La Paz: Fundemos. 277-328. 
UGGLA, Fredrik. 2009. "Bolivia: un año de vivir peligrosamente". Revista de Ciencia Política 29 (2): 247-273.

UN (Unidad Nacional). 2005. Dar la cara por Bolivia. Acciones de gobierno 20062010.

[http://www.oep.org.bo/proces_electoral/generales2005/comunicacion/egp 2005_programa_un.pdf] página descargada el 10 de noviembre 2016.

\section{$(\infty))_{\text {EY }}$}

\section{This journal is published by the University Library System of the University of Pittsburgh as part of its D-Scribe Digital Publishing Program, and is cosponsored by the University of Pittsburgh Press.}

